\title{
Schematic exemplars as items in multiple-item recognition learning
}

\author{
DONALD H. KAUSLER, LAURA L. MAJCHER, and JERRY N. CONOVER \\ University of Missouri, Columbia, Missouri 65201
}

\begin{abstract}
Schematically related dot patterns (i.e., distortions of a common prototype) were employed as either the right items or the wrong items of a multiple-item recognition learning task. Relative to a control list containing unrelated items, the presence of related right items yielded facilitated performance, but only early in practice. By contrast, the presence of related wrong items yielded an inhibitory effect early in practice. The results suggested that shared features are more likely to be extracted from the information present in right items than from the information present in wrong items.
\end{abstract}

Multiple-item recognition learning ${ }^{1}$ has recently been riewed from the perspective of information processing theory (Kausler, Pavur, \& Yadrick, 1975; Kausler. Note 2). According to this conceptualization, right item members of study pairs are processed at a deeper level than are wrong item members. Sensory and/or semantic features are extracted from right items during study phases. Representatives of these features in the subject's permanent memory are the recipients of response-based frequency units (Kausler. 1974). By contrast, wrong items are likely to be processed wholistically, that is, as undifferentiated entities. Preliminary evidence for this hypothesis was provided by Kausler et al. (1975). Homophones of prior right items were more likely to be falsely identified as old items than were homophones of prior wrong items. Presumably, phonemic features were extracted to a greater extent from right items than from wrong items, thereby generating the greater false recognition for new test items that contained identical phonemic features of prior right items.

An extension of the preceding analysis is possible when study items themselves overlap featurally. If the overlap rests in right items. the shared features are likely to be extracted, thus providing a means of short-circuiting the rote acquisition of the study list. During a test phase, right items become identifiable by the presence of these previously tagged common features. The net effect should be facilitation of applying frequency theory's Rule 1 (always select pairwise right items; Ekstrand, Wallace, \& Underwood, 1966). However, the shared features are less likely to be extracted when the overlap rests in wrong items. Thus, there should be little facilitation via the application of frequency theory's Rule 2 (always avoid selecting pairwise wrong items; Ekstrand et al.. 1966). Evidence for this differential mediating process was obtained by Kausler, Erber, and Olson (1970). They found that acquisition was

This research was supported in part by National Science Foundation Grant BMS75-05007 awarded to the first author. facilitated significantly when the right items of their list were instances of common taxonomic categories. On the other hand, acquisition was facilitated only slightly when the wrong items were instances of the same taxonomic categories. In this case, the extracted shared feature was semantic in nature (i.e.. categorical membership).

The present study offered a further test of the differential feature extraction hypothesis. Feature overlap in the present case was manipulated through the use of exemplars of a dot pattern prototype (Posner, Goldsmith, \& Welton, 1967) as right or wrong items. That is, the items of a given pairwise function were all 5-bit distortions of the same schematic prototype. A preliminary study indicated a slight facilitating effect-but only early in practice-for the right items locus and a slight inhibitory effect-but, again, only early in practicefor the wrong item locus. The subjects in this preliminary study were not instructed as to the rules employed to generate right/wrong items. In the hope of stimulating a more effective feature-extraction process, subjects in the present study were fully informed as to the nature of their right and wrong items.

\section{METHOD}

\section{Subjects and Design}

The subjects were 30 students in a large introductory psychology class who fulfilled a course requirement by their participation in the present experiment. They were assigned randomly and equally to three groups: Group $\mathbf{R}$ (distortions of a common dot prototype served as right items); Group W (the same distortions served as wrong items); and Group C (a control group in which neither right nor wrong items were related patterns).

\section{Materials}

Twelve random patterns, or prototypes, of nine dots each were constructed according to the rules employed by Posner et al. (1967). Two of the patterns, Prototypes 1 and 2, were randomly selected to serve as experimental schema in the preliminary experiment, one for half of the subjects and the other for the remaining half. Effects attributable to schematic variation were negligible. Consequently, only Prototype 1 served as the experimental schema for the study reported here. Six 5-bit
} 
Table 1

Summary Statistics for Errors With Instructed Subjects

\begin{tabular}{lrlrccccr} 
& \multicolumn{2}{c}{ Block 1 } & \multicolumn{2}{c}{ Block 2 } & \multicolumn{2}{c}{ Block 3 } & \multicolumn{2}{c}{ Total } \\
& M & SD & M & SD & M & SD & M & SD \\
\hline Group R & 6.50 & 3.98 & 5.00 & 4.80 & 4.40 & 4.53 & 15.90 & 11.45 \\
Group W & 10.60 & 4.48 & 4.60 & 4.84 & 3.60 & 5.72 & 18.80 & 12.95 \\
Group C & 8.20 & 2.40 & 5.10 & 3.07 & 4.10 & 3.38 & 17.40 & 6.28 \\
\hline
\end{tabular}

distortions were derived from this prototype. The distortion functioned as the right items for Group $\mathrm{R}$ and the wrong items for Group W. Prototypes 3-8 were randomly selected from the remaining prototypes to serve as wrong items for Group $\mathbf{R}$ and right items for Group W. For both groups, right-wrong pairings were determined by random assignment. Half of the subjects in Group C had Prototypes 3-8 function as right items and 1,2 , and 9-12 function as wrong items. The remaining subjects in Group C had the right-wrong roles reversed. The two subgroups of Group C did not differ substantially in performance and were pooled for the analyses reported later.

A possible complication with these materials is an unintentional differential in similarity between right-wrong dot patterns used for Groups $\mathrm{R}$ and $\mathrm{W}$, relative to those used for Group C. That is, if the similarity between paired items is greater when related distortions make up half of each pair than it is when unrelated items compose all pairs, the positive effect of feature extraction for Groups R and W could be mitigated. Conversely, if the similarity is less, the positive effect could be attributable to the greater ease of intrapair discriminability, independently of shared feature extraction and subsequent rule application. As a check of this possibility, all pairs employed in this study were rated for degree of similarity on a 7-point scale $(1=$ very low similarity; $7=$ very high similarity $)$ by a separate group of eight subjects. The mean ratings for the pairs entering into Groups R-W vs. Group C were quite comparable, each averaging approximately 2.6. In addition, the variability between set members was roughly equal across all conditions. Thus, it seems unlikely that differential intrapair similarity was a confounding factor in the present study.

\section{Procedure}

Practice was by the study-test method. On each of 20 study phases, right-wrong pairs were exposed via a slide projector for $2 \mathrm{sec}$. The items were viewed as top-bottom pairs, with the right member of each pair labeled "correct" and the wrong member labeled "incorrect." On each of 20 test phases, the pairs were exposed for $4 \mathrm{sec}$ without labels, and subjects identified their right item choices on an answer sheet by writing $T$ or $B$. The exposure durations were determined after extensive pilot research with various durations. The objectives were to find durations that would yield rates of acquisition consistent with those generally found with linguistic elements in multiple-item recognition learning. Standard control procedures were employed to eliminate confounding from either serial order or spatial position cues. The subjects in all groups were told in advance about the nature of their items, including, for Groups $\mathrm{R}$ and $\mathrm{W}$, the general method used to construct distortions from a common prototype.

Finally, the subjects in Groups $\mathrm{R}$ and $\mathrm{W}$ were given a transfer test following the last study-test phase. For this test, the subjects were shown a pair of items, one of which was the experimental schema (Prototype 1) and the other an irrelevant prototype that had not entered into their list materials. They were then asked to select what hey perceived to be the relevant prototype for the prior session.

\section{RESULTS AND DISCUSSION}

Errorless performance was attained by most subjects in all three groups after 12 trials.
Consequently, analysis was restricted to errors over Trials 1-12. Initially, trials were grouped into blocks of four trials each. Summary statistics for total errors and errors per block are given in Table 1 .

The main effect for trial blocks was highly significant, $F(2,54)=20.37, p<.001$, and the main effect for groups fell far short of significance, $F(2,27)<1$. The Groups by Blocks interaction effect approached significance, $F(4,54)=2.33, \mathrm{p}<.10$. In addition, the linear component of the interaction effect was significant, $F(2,54)=3.78, p<.05$. It may be seen from Table 1 that the difference in linear trends between groups resulted from differential proficiency levels early in practice, followed by virtually identical performance levels thereafter. The between-group effect approached significance for the tirst block of trials, $F(2,27)=3.00, p<.10$. Only the Group R vs. Group W contrast was significant for this block. Duncan's test. $p<.05$. The betweengroup effect was clearly nonsignificant for the third block of trials. $F(2,27)<1$.

Group differences on the early trials were probed further. Means and standard deviations for errors on Trials 1 and 2 combined were 2.90 and 2.23, 6.40 and 2.63. and 4.90 and 2.18 for Groups $R, W$, and $C$. respectively. The between-group effect was significant. $F(2.27)=5.55, p<.01$. A Duncan's test revealed that the contrast between Groups $R$ and $W$ was significant, $p<.01$, and the contrasts between Groups $R$ and $C$ and Groups $W$ and $C$ approached significance, $p<.10$. A trial by trial analysis demonstrated that only Group $\mathrm{R}$ exceeded the chance performance level on Trial 1 (mean errors $=1.60$; correlated-t $(9)=3.41, p<.01$ ). Performance did not exceed the chance level for Group W until Trial 3 (mean errors $=3.40,3.00$, and 1.80 on Trials 1,2 , and 3 , respectively). Group C's performance approached an above-chance level on Trial 2, correlated-t(9) = $1.88 . \mathrm{p}<.10$ (mean errors $=2.80,2.10$, and 1.80 on Trials 1.2. and 3. respectively). The pattern of results corresponds closely to that of the preliminary experiment. Asymmetry was again found for Groups $R$ and $W$, with Group $R$ displaying an early facilitation in performance and Group $W$ an early inhibition in performance.

Finally, the results of the transfer test were indicative of differential degrees of featural processing by Groups R and W. All 10 of the subjects in Group R selected the appropriate schema on the transfer test, as compared to only 4 of the 10 subjects in Group W, $\chi^{2}(1)=5.95, p<.02$. Thus, there is good reason to believe that the subjects in Group $R$ extracted sufficient features shared by the right items to mediate recognition of the underlying prototype. The failure to demonstrate a more pronounced positive effect of this extraction process on intrapair discriminations probably resulted from the deviations of subject-constructed schema from the formal experimental prototype. That is, a subjective schema 
may be an impoverished one that incorporates only a few features that were suggested by several right items. These features are sufficient to mediate recognition of both the relevant right items and the experimental prototype that share them. Presumably, it was these right items that produced the early facilitation for Group $R$ in both experiments. However, the same features are sufficiently distorted in the remaining right items to prohibit ready recognition of these items as derivatives of the subjective schema. Consequently, intrapair discriminations involving these remaining right items must be based on rotely accrued frequency units.

There is little evidence to suggest that the subjects in Group W constructed subjective schema from extracted features, even when instructed as to their availability for extraction. The nature of the multiple-item task is such that it directs attention mainly to right items. The early inhibitory effect for Group W most likely reflects abortive attempts to accomplish this extraction, with consequent diminished attention being given to right items. Such attempts are abandoned after a few trials on the task.

\section{REFERENCE NOTE}

1. Kausler, D. H. Differential processing of correct and incorrect items in multiple-item recognition learning. Invited paper. Midwestern Psychological Association, Chicago, Illinois, 1975.

\section{REFERENCES}

Ekstrand, B. R., Wallace, W. P., \& Underwood, B. J. A frequency theory of verbal discrimination learning. Psychological Review, 1966, 73, 566-578.

KaUSler, D. H. Continuity of processes across variants of recognition learning. In R. L. Solso (Ed.), Theories of cognitive psychology: The Loyola Symposium. Potomac, Maryland: Erlbaum, 1974.

Kausler, D. H., Erber, J. T., \& Olson, G. A. Taxonomic instances as right or wrong items and selection strategies in verbal discrimination learning. American Journal of Psychology, 1970, 83, 428-435.

Kausler, D. H., Pavur, E. J., JR., \& Yadrick, R. M. Single-item recognition following a verbal discrimination study trial. Memory \& Cognition, 1975, 3, 135-139.

Posner, M. I., Goldsmith, R., \& Welton, K. E., JR. Perceived distance and the classification of distorted patterns. Journal of Experimental Psychology, 1967, 73. 28-38.

\section{NOTE}

1. This variant of recognition learning is commonly called verbal discrimination. Since the to-be-discriminated items need not involve linguistic elements, the generic term of multipleitem recognition learning seems more appropriate for the present study.

(Received for publication July 14, 1975.) 